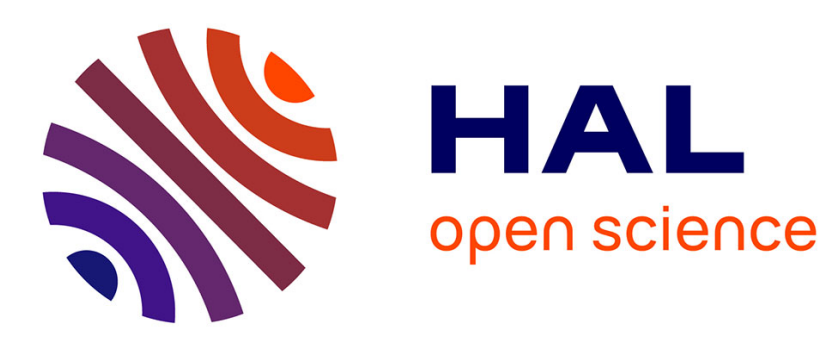

\title{
Optimal Sinks Deployment and Packet Scheduling for Wireless Sensor Networks
}

\author{
Nadjib Achir, Paul Mühlethaler
}

\section{To cite this version:}

Nadjib Achir, Paul Mühlethaler. Optimal Sinks Deployment and Packet Scheduling for Wireless Sensor Networks. Wireless Days, Nov 2014, Rio de Janeiro, Brazil. hal-01092543

HAL Id: hal-01092543

\section{https://hal.inria.fr/hal-01092543}

Submitted on 8 Dec 2014

HAL is a multi-disciplinary open access archive for the deposit and dissemination of scientific research documents, whether they are published or not. The documents may come from teaching and research institutions in France or abroad, or from public or private research centers.
L'archive ouverte pluridisciplinaire HAL, est destinée au dépôt et à la diffusion de documents scientifiques de niveau recherche, publiés ou non, émanant des établissements d'enseignement et de recherche français ou étrangers, des laboratoires publics ou privés. 


\section{Optimal Sinks Deployment and Packet Scheduling for Wireless Sensor Networks}

\author{
Nadjib Achir \\ Universit Paris 13, Sorbonne Paris Cit, \\ L2TI (EA 3043) \\ F-93430, Villetaneuse, France \\ Email: nadjib.achir@univ-paris13.fr
}

\author{
Paul Muhlethaler \\ INRIA HiPERCOM, Rocquencourt \\ B.P. 105, 78153 Le Chesnay, France \\ Email: paul.muhlethaler@inria.fr
}

\begin{abstract}
In this paper we propose an optimal deployment and distributed packet scheduling of multi-sink Wireless Sensors networks (WNSs). This work is devoted to computing the optimal deployment of sinks for a given maximum number of hops between nodes and sinks. We also propose an optimal distributed packet scheduling in order to estimate the minimum energy consumption. We consider the energy consumed due to reporting, forwarding and overhearing. In contrast to reporting and forwarding, the energy used in overhearing is difficult to estimate because it is dependent on the packet scheduling. In this case, we determine the lower-bound of overhearing, based on an optimal distributed packet scheduling formulation. We also propose another estimation of the lower-bound in order to simulate non interfering parallel transmissions which is more tractable in large networks. We note that overhearing largely predominates in energy consumption. A large part of the optimizations and computations carried out in this paper are obtained using ILP formalization.
\end{abstract}

\section{INTRODUCTION}

The monitoring and the control of physical environments is becoming a major feature of the technology landscape. Consequently, a large number of companies are proposing new small devices which can monitor various physical parameters such as temperature, humidity, vibration, pressure,etc. These devices, called sensors, can be deployed to build a Wireless Sensor Network (WSN). In this case, in addition to the detection capability, the sensors should be able to communicate between each other in order to propagate event notifications. Basically, when an event is detected, each sensor sends a message to one specific base station. This station called the "sink" is in charge informing the user-application that an event has occurred. Over the last decade, Wireless Sensor Networks (WSN) have generated considerable enthusiasm among the networking research community. Many studies have been carried in order to apply WSN to a wide range of applications, such as: environmental monitoring, military target tracking, weather forecast, home automation, intrusion detection, etc [1].

Recent research in WSN has mainly focused on several networking issues, such as routing, MAC, data gathering and dissemination mechanisms. Nevertheless, few studies have been achieved regarding the WSN deployment problem. Among existing studies achieved regarding the wireless sensor network deployment problem, the majority are focusing on the problem of minimizing the number of deployed sensors while maximizing the area coverage (also referred as quality of monitoring). The area coverage can be defined as the percentage of the area, or a finite set of points within the area, in which an event can be monitored by at least one sensor (expressed as 1-coverage). A more general definition was proposed in the literature (denoted as $k$-coverage), in which the objective is to achieve more redundancy and reliability, and thus requires that each point within the region of interest is monitored by at least $k$ sensors. On the other hand, other works focused on the problem of sinks deployment. Some of the proposed solutions compute the optimal sinks locations using optimal formalization. However, the majority of the solutions proposed aim to incorporate energy or delay in the optimization problem [2] [3], which highly reduce the scale of the considered problems. We believe that a simple formalization of the problem based only on the number of hops could provide good performances. Other approaches, use iterative techniques. In [4], the locations of the sink nodes are found using the well known k-means clustering algorithm. Finally, some of the previous studies assume mobile sinks [5] [6]. The main weakness of this approach is that sinks movement is not considered for the energy consumption computation.

The performance metric that is most often considered in WSN deployment is the network lifetime. Indeed, a WSN can only fulfill its purpose as long as it is considered as "alive". It is therefore an indicator for the maximum utility a sensor network can provide. In general way, network lifetime can be defined as the time span from the deployment of the wireless sensor network until the time where the network can be considered as inoperative, which strongly dependent on the targeted application. The problem of maximizing network lifetime has been largely addressed in many works with different approaches, such as: energy based routing protocols, energy-based MAC protocols, etc. However, few studies were conducted on enhancing energy consumption only by finding the most appropriated topology [7], [8].

In this paper, we are interested in minimizing the energy consumption of a wireless sensor network, when multiple sinks are deployed in the area. It is clear that the energy cost is highly dependent on the topology of the network, which itself depends on the localization and the number of 
sinks deployed. Thus, we start this work by computing the optimal number of sinks that should be deployed according to a given maximum number of hops allowed in the area. This is done by formulating the problem as a set-covering problem. It is clear that this problem is NP-hard, however we have been able to resolve the problem for quite a large number of sensors. After deploying the sinks, we propose a computation model of the energy consumed by each sensor in the network. In addition to the energy consumed in the Idle mode, we consider both the energy consumption caused by packets reporting, forwarding and finally, overhearing. The main difficulty is related to the overhearing mode, as for the packet reporting and forwarding, the energy can be deduced analytically, based on the topology of the network. However, for the overhearing the amount of energy consumed by each node greatly depends on the packet scheduling in the network. We propose to derive the lower bound of the energy consumed due to the overhearing phenomenon. This lower bound is difficult to estimate. To overcome this difficulty, we propose a distributed packet scheduling algorithm based on an ILP formalization of the problem.

The remainder of this paper is organized as follows: In section II we define our network model, including the targeted application and the medium access control protocol. In section III we show how we compute the optimal number of sinks that should be deployed according to a given maximum number of hops allowed in the area. In section IV we detail the energy consumption computation. The results are given in section V. Finally, in section VI we conclude the paper, and we suggest future research directions.

\section{NETWORK MODEL}

In this paper, we consider that we have access to a $2 \mathrm{D}$ geographical map denoted as $A$. For simplicity, but without loss of generality, we suppose that $A$ is a square area. In order to reduce the computational complexity of the problem, this area is discretized into cells of square units. A unit is defined as a normalized physical distance. Thus, the area $A$ is a square with a side equal to $n$ units. To simplify the analysis, in the rest of the paper we refer to each square unit (cell) of $A$ by its barycenter point. In other words, when a sensor is deployed at point $p \in A$ then this means that a sensor is placed at the barycenter of the cell containing $p$. Moreover, we consider that a set of connected sensors, denoted by $S$, is already deployed within $A$ and according to homogeneous Poisson point process [9] [10]. In addition to the sensors, we assume that a set of sinks, denoted by $K$, are also deployed in $A$, in such a way that each sensor is at most $h$ hops from the nearest sink. The number and the position of the sinks are given in the next section. In addition, we consider a reporting application, where the sensor nodes sense their environment and report it to the nearest sink, using a shortest path as routing strategy. We assume that all the sensors do the sensing process simultaneously and periodically. We denote this period by $T$, which defines the interval of time separating two reporting packets. Finally, we also consider that all the reporting packets for all the sensors have the same size denoted by $d$. Finally, to avoid collisions, we assume Time Division Multiple Access (TDMA) as medium access control protocol. The wireless channel is subdivided into slots, and each sensor can send only one packet within a slot. We also introduce the notion of round, denoted by $R$, as the period of time where the reporting packets generated by all the sensors are received by the sink. For the simplicity of the analysis we consider that $T \geq R$.

\section{Sink Deployment PRocedure}

As mentioned above, our first objective is to deploy the minimum number of sinks, denoted as $K^{*}$, in such a way that for each sensor $s$ there a sink $k$, where the distance in terms of the minimum number of hops to reach $s$ from $k$ is less than or equal to $h$. This problem can be formulated as a set covering problem and could be defined as follows: Given a finite set of points $\Omega$, the main purpose is to find a family $S=\left\{s_{1}, s_{2}, . ., s_{N}\right\}$ of subsets of $\Omega$ (i.e. $s_{i} \in \Omega, j=$ $\{1, \ldots, N\})$ with a minimum cardinality such that $\bigcup_{\forall i} s_{i}=\Omega$. One classical approach to resolving this problem is to use an Integer Linear Program - ILP formulation. In this case the problem can be formulated as follows:

$$
\begin{array}{ll}
\operatorname{minimize} & \sum_{1 \leq j \leq N} x_{j} \\
\text { subject to: } & \sum_{1 \leq j \leq N} a_{i j} x_{j} \geq 1, \forall i \in \Omega \\
& x_{j} \in\{0,1\}, \forall j \in\{1, . ., N\}
\end{array}
$$

where $x_{j}$ and $a_{i j}$ are zero-one variables. The decision variable $x_{j}$ is equal to 1 , if the subset $s_{j}$ is chosen in the optimal solution, 0 otherwise and $a_{i j}$ is equal to 1 , if the point $i$ is covered by the subset $s_{j}, 0$ otherwise.

Adapting this last formulation to our problem implies that the set $\Omega$ corresponds to the set of sensors $S$ already deployed in the targeted area. The variable $x_{j}, j \in\{1, . ., n \times n\}$, is a binary variable that indicates whether a sink is deployed at point $j$. Precisely, $x_{j}$ is equal to 1 if and only if a sink is positioned at point $j, 0$ otherwise. And finally, each variable $a_{i j}, \forall i \in S$ and $\forall j \in\{1, . ., n \times n\}$, is equal to 1 if the sensor $i$ is within the $h$ hop coverage area of a sink positioned at the point $j$. In order to compute the distance in terms of hops between a sensor $i$ and a sink deployed at point $j$, we apply the shortest path strategy for the undirected graph $G_{j}=\left(V_{j}, E_{j}\right)$, where $V_{j}$ is a set of vertices including all the sensors and the sink positioned at $j$ (i.e. $V_{j}=S \cup\{j\}$ ) and $E_{j}$ is the set of undirected edges denoting the possibility of a communication link between nodes. More precisely, we consider the existence of a link between two nodes, one of which may be the sink, if and only if both nodes are within the transmission range of each other. In the following, we consider that all the network nodes have the same transmission range denoted by $r$. Given the parameters defined above, the multi-sink deployment problem can be defined as following: 


$$
\begin{array}{ll}
\text { minimize } & \sum_{\substack{1 \leq j \leq n \times n \\
\text { subject to: }}} \sum_{\substack{1 \leq j \leq n \times n\\
}} a_{i j} x_{j} \geq 1, \forall i \in S \\
& i \neq j, \\
& x_{j} \in\{0,1\}, j=1, \ldots, n \times n
\end{array}
$$

Based on this last formulation, we can derive the optimal set $K^{*}$ of sinks that should be deployed in order to guarantee that each node is at most $h$ hops from a sink. Thus, we can model the underlying network as an undirected graph $G=(V, E)$, where each network node (i.e. sensor or sink) is represented by a vertex $v \in V$ (where $V=K^{*} \cup S$ ) and $E$ is the set of undirected edges denoting the communication links. From the previous graph $G$ we derive a new graph $G^{\prime}=\left(V^{\prime}, E^{\prime}\right)$, by adding a new virtual vertex representing a single super sink which is directly connected to all the sinks belonging to the set $K^{*}$. Thus, $V^{\prime}=V \cup\left\{k^{\prime}\right\}$ and $E^{\prime}=E \cup\left\{\left(n, k^{\prime}\right) \mid \forall n \in K^{*}\right\}$. The main idea behind this graph transformation is to be able to derive the routing strategy adopted in our network by applying a classical Shortest Path Tree or a Minimum Spanning Tree algorithm, which require a single source. Since we fix the same weight for all the edges, thus computing the shortest path from any of the network sensors to the closest sink leads to computing the shortest path from the same sensor to the super sink. From these considerations, we can then define the set $P$ as the set of shortest paths for all $v$ in $\left\{V-K^{*}\right\}$. We also define $P(v)$ and $P(e)$ as the subsets of $P$, where the node $v$, respectively the edge $e$ is present.

\section{ENERGY COMPUTATION}

In a general way, network lifetime can be defined as the time span from the deployment of the wireless sensor network until the time where the network becomes inoperative. It is clear that considering a network to be inoperative is strongly dependent on the targeted application. For example, one can consider the percentage or the number of alive nodes, the area coverage, the connectivity of the network, or some specific applicationbased quality of service requirements. In this paper, we assume that the sensor network is considered to be inoperative when the first sensor runs out of energy. As we are considering a reporting application, we can make the assumption that the sensor energy consumption is mainly affected by the communications function, since the energy consumed by the CPU is relatively the same for all nodes and can be considered to be constant. Finally, in order to compute the network lifetime, we consider that the energy consumption at each node can be computed as the sum of the energy used when of: 1.) Periodic packet reporting, 2.) Packet forwarding, 3.) Packet overhearing and finally, 4.) Idle mode. In the following we detail each case independently.

\section{A. Reporting Energy computation}

In this paper, we consider in this paper, that each sensor periodically sends packets to the sink. The amount of energy consumed by the sensor is dependent to the sampling process and the transmission of the sampled data. The energy consumed by a sensor $v$, for reporting each measurement can be formulated as follows:

$$
E_{\text {reporting }}(v)=E_{\text {sampling }}+E_{t x}(d)
$$

where $d$ is the packet size, $E_{\text {sampling }}$ is the energy used for event detection and finally $E_{t x}$ is the energy consumed due to the packet transmission. In the following, we assume that the sampling energy is negligible compared to the transmission energy (i.e. $E_{\text {sampling }}=0$ ) and that all the sensors generate packets of the same size.

\section{B. Forwarding Energy computation}

The packet forwarding procedure is divided into two phases: a first phase of packet reception and a second phase of packet transmission, after identifying the next hop. In this case, the forwarding energy consumption for a given node $v$ is equal to the energy spent for receiving a packet plus the energy spent for sending a packet that we multiply by the number of paths where the node $v$ is present, exempt the path where the node $v$ is as a source. Formally, $E_{\text {forwarding }}(v)$ can be formulated as follows:

$$
E_{\text {forwarding }}(v)=\sum_{i=1}^{|P(v)|-1}\left(E_{r x}(d)+E_{t x}(d)\right)
$$

where $E_{r x}(d)$ corresponds to the energy consumed by a sensor for the reception of a packet of size $d . P(v)$ is the subsets of $P$, where the node $v$ is present.

\section{Overhearing Energy computation}

In addition to the packet reporting and the forwarding procedure, sensor nodes may see their energy decreasing because of the overhearing phenomenon. Because of the broadcast nature of wireless channels, sensors may overhear the packet sent by other sensors in their vicinity even if they are not the intended recipients of these packets. Unfortunately, this packet overhearing can result in unnecessary expenditure of battery energy. One solution to decrease the overhearing impact is to consider sensors with the ability of on-the-fly header packet decoding. This mechanism allows a sensor to know if it is the intended destination of the packet and thus shutting the radio off if not. This mechanism requires sophisticated electronics which leads to high prices and complexity in current market sensors. In this paper, we take into consideration the overhearing phenomenon and we model the energy consumed by each node. More precisely, we derive the lower bound of energy consumed due to overhearing.

The lower bound corresponds to the optimistic case, by achieving the maximum number of parallel communication allowed. Indeed, since overhearing is due to the fact that a sensor can hear other sensor transmissions, one way to reduce the overhearing effect is to maximize parallel packet transmissions and thus reduce the period of overhearing for each sensor. This leads to computing the lower bound overhearing energy consumptions for each sensor. In the following, we present an 
Integer Linear Programming formulation of the lower bound computation problem of the overhearing effects. Our problem can be formulated as an integrated task scheduling problem, where each packet transmission can be considered as a task and our objective is to minimize the overall completion time of all the tasks.

We start by considering $K$ sinks deployed in the area. The set $V$ can be subdivided into $K$ independent sets (i.e. clusters) of a given number of nodes, such as $V^{1} \cup V^{2} \cup \cdots V^{k} \cdots \cup$ $V^{K}=V$. In what follows, we sometimes use the notation $u^{k}$ for a node $u$ that is associated to the sink $k$. We consider the binary variable $s_{t}$, that identifies the usage of the slot $t$. More precisely, $s_{t}$ is equal to 1 if a packet is transmitted during the slot $t$ and 0 otherwise. Similarly, we define $f_{t}(u, v)$ as a binary variable that represents the state of the directed link $(u, v)$ at slot $t$. In this case, $f_{t}(u, v)$ is equal to 1 , if the node $u$ sends a packet to node $v$ in slot $t$, and 0 otherwise. We also define $p_{t}(v)$ as the number of packets pending at node $v$ at the end of slot $t$. Finally, we consider $T_{\max }$ as the maximum number of time slots to complete the transmission of all packets of all the sensors to the sink. Accordingly, we can formulate our problem as follows:

$$
\begin{aligned}
& \text { Min } \sum_{1 \leq t \leq T_{\max }} s_{t} \\
& \text { s.t. } \quad p_{0}(u)=n_{u} ; \forall u \in V \\
& p_{t}(u) \geq 0 ; \forall u \in V, 1 \leq t \leq T_{\max } \\
& p_{t+1}(u)=p_{t}(u)-\sum_{v \in V} f_{t}(u, v) \\
& +\sum_{v \in V} f_{t}(v, u) ; \forall u \in V, 1 \leq t \leq T_{\max } \\
& \sum_{u \in V^{k}} p_{0}(u)=\sum_{1 \leq t \leq T_{\max }} \sum_{u \in V^{k}} f_{t}(u, k) ; \forall k \in K(5 \mathrm{e}) \\
& \sum_{v \in V} f_{t}(u, v) \leq p_{t}(u) ; \forall u \in V, 1 \leq t \leq T_{\max } \\
& \sum_{v \in V} f_{t}(u, v) \leq 1 ; \forall u \in V, 1 \leq t \leq T_{\max } \\
& f_{t}(u, v)+f_{t}(v, u) \leq 1 ; \\
& \forall u, v \in V, 1 \leq t \leq T_{\max } \\
& \sum_{v \in V} \sum_{(w, z) \in E}\left(\left(f_{t}(u, v)+f_{t}(w, z)\right)\right. \\
& \left.\cdot \mathbb{1}_{z \in N(u)}\right) \leq 1 ; \forall u \in V, 1 \leq t \leq T_{\max } \\
& f_{t}(u, v) \leq s_{t} ; \forall(u, v) \in E, 1 \leq t \leq T_{\max } \\
& s_{t+1} \leq s_{t} ; 1 \leq t \leq T_{\max }
\end{aligned}
$$

As indicated in the problem formalization, the objective is to minimize $\sum_{1 \leq t \leq T_{\max }} s_{t}$, which identifies the total number of time slots required to complete the delivery of all packets to the sinks. The first two constraints initialize the number of packets generated by each sensor $u$ to $n_{u}$ and guarantees that this number can never be less than zero. The next constraint (5d) is for flow conservation. More precisely, this constraint guarantees that the number of packets present at a node for a given time $t$ slot is equal to the number of packets pending at the end of the previous time slot, to which we add/subtract packet sent/received to/from the neighbor nodes. The constraint (5e) ensures that all packets generated by a node are delivered to the corresponding sink. The constraint (5f) states that a node $u$ can send a packet if and only if there is more than one packet pending at the node $u$. The constraint $(5 \mathrm{~g})$ states that a node transmits to at most one neighbor, and the next constraint restricts nodes from both transmitting and receiving packets within the same slot. Similarly, the constraint (5i) indicates that at most one neighbor of a node can transmit in a slot. This will guarantee that two nodes, which are within overhearing range of each other, cannot use the same slot. The constraint (5j) states that if the slot $t$ is used, this means that at least one link is active. Finally, the last constraint guarantees that the slots used are contiguous. The resolution of the last problem provides the minimum number of slots in order to complete the packet delivery from the sensors to the sinks. Moreover, we can derive, for each slot, which node is sending a message, which node is receiving this message and finally, which nodes are overhearing it.

\section{Idle Mode Energy computation}

Finally, the last mode that we consider in this paper is the idle mode. In this state, the sensors have to continue listening to the wireless channel by keeping their radios in receive mode, which is also energy consuming. Since, we know the reporting period $R$, and according to the above formulations where we derive the periods where the node is sending, receiving and overhearing packets, thus we can easily deduce the portion of time where the state of the sensor is idle.

\section{REsults}

We start by evaluating the optimal sinks deployment procedure that we propose in section III . The main objective is to evaluate the ILP limitations in terms of scalability. In Figure 1 we show the sinks deployment for an homogeneous Poisson point process with an average of $\lambda=300$ sensors on a tore of $n \times n(n=50)$ and for a maximum number of hops equal $(h)$ to 5 . The optimal number of sinks obtained is equal to 5 sinks. We can notice from this first result that the ILP scheme that we use to compute the deployment is efficient to find an optimal solution. Indeed, we were able to compute the solution for $\lambda=300$ sensors and for a number of possible sink locations of $\left(n^{2}-\lambda\right)$. The average time required to perform this computation on a standard PC is shown in Figure 2; we notice that this time is a decreasing function of $h$. In fact the problem complexity depends more on the area size than the number of sensors. Taking only the sensor locations as possible sink positions could further reduce this complexity.

Figure 3 provides the average number of sinks versus the maximum number of hops $h$. We observe that this number is very stable as shown by the error-bars. This number is decreasing more quickly than $\frac{1}{h}$ and less quickly than $\frac{1}{2^{h}}$. 


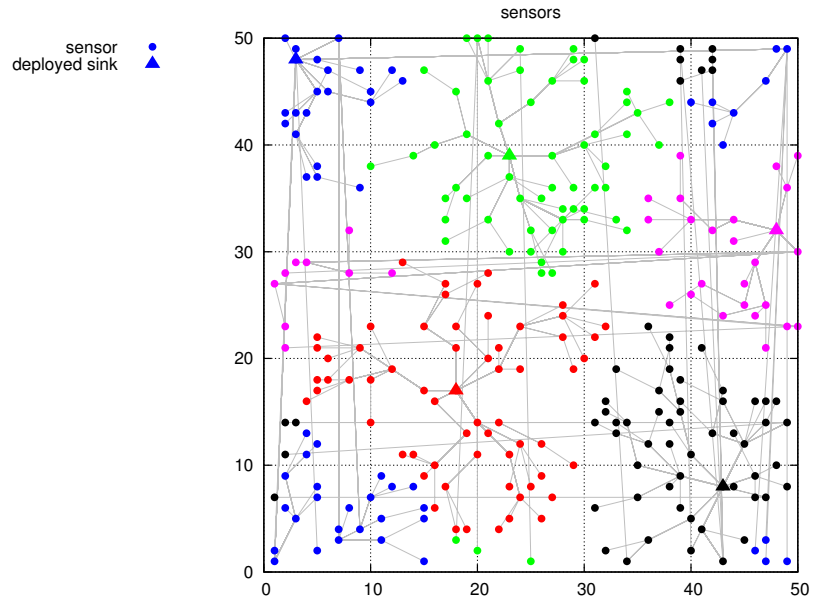

Fig. 1. Sink deployment for and area of $50 \times 50 \quad(\lambda=300$ sensors $)$

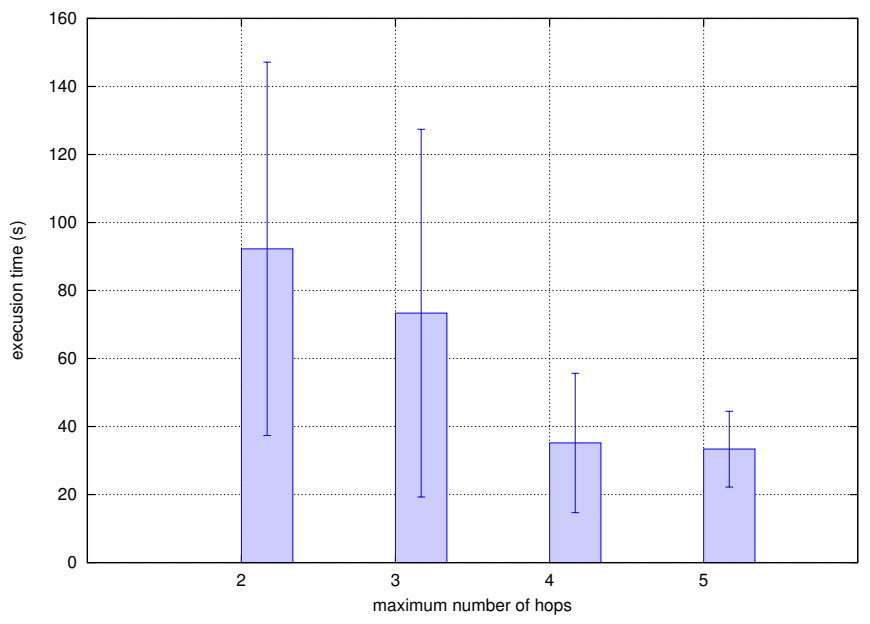

Fig. 2. Execution times vs maximum hops

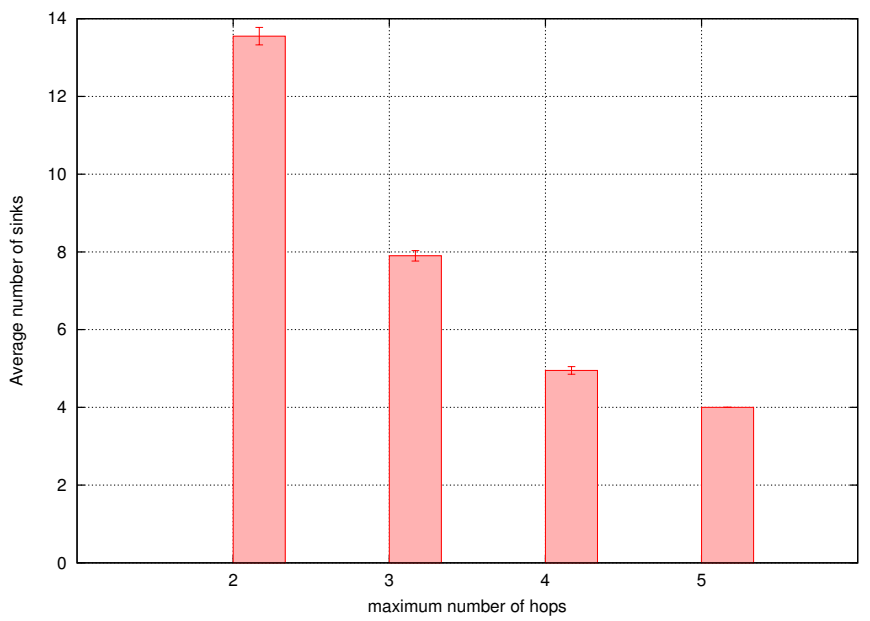

Fig. 3. Average number of deployed sinks vs maximum hops
After deploying the sinks, we start the investigation of the energy consumption. In the following, we consider the sensors energy consumption values of the CC2420 family [11], which have been used in many sensor platforms. We assume that the transmission power is independent on the path loss. Figure 4 shows the energy consumption in a small network of 16 sensors with only one sink in the center and for a maximum number of hops is equal to 2 . The choice of a small network is justified by the computational complexity of estimating the lower bound of the energy used in overhearing. Indeed, the optimization problem proposed in the last section is not scalable in nature and could be solved only for small scale network. As shown in figure 4, overhearing predominates in the energy consumption. In addition the the lower bound, we also computed the upper bound. The upper bound corresponds to the case where each sensor listens to the transmission of all packets in its neighborhood. The upper and lower bounds, which are close, demonstrate that, even in the best cases, overhearing is by far the most energy consuming process in the network.

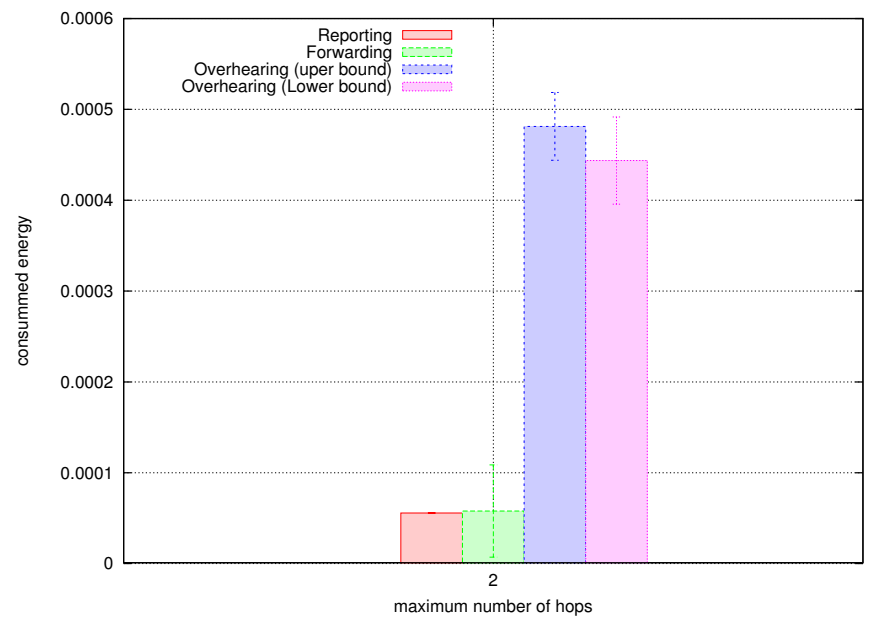

Fig. 4. Reporting, Forwarding and Overhearing (upper bound/Lower bound) energy consumption

Finally, figure 5 illustrates the energy consumption in a larger network $(\lambda=300$ sensors). We have presented an upper bound for the overhearing and another estimation of lower-bound overhearing based on simple graph coloring techniques. The main idea is to assign colors to the nodes (sensors) of the overhearing graph so that no two adjacent nodes have the same color. In other words, two nodes with the same color can transmit data during the same slot. In this case, we could increase the number of parallel packet transmissions. We assume that the amount of overhearing in a real network would be between these two values. Here again we note that overhearing predominates in the energy consumption.

\section{CONCLUSION}

In this paper, we propose focuses on multi-sink Wireless Sensors networks deployment and packet scheduling in order to reduce the energy consumption related to the overhearing. 


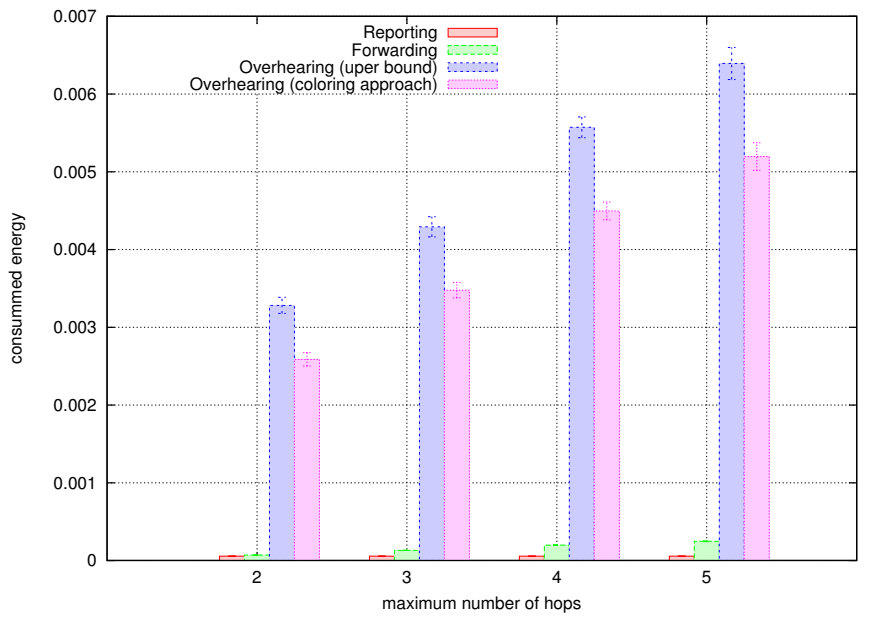

Fig. 5. Reporting, Forwarding and Overhearing (upper bound) energy consumtpion vs maximum hops

The main objective is to be able, for a given number of sensors and a given maximum number of towered hops, to: i.) Compute the optimal number of sinks to deploy and $i i$.) Estimate the lower-bound energy consumption. We formulate the problem of deployment of an optimal number of sinks as an ILP problem. The results obtained show that this approach could be used for a relatively large number of sensors. Then, we estimate the energy consumption of the network obtained. We focuses our analysis on the computation of lower and upper bounds for overhearing which is by far the most energy consuming process. Unlike the upper bound, which is easy to derive, the lower bound is difficult to estimate. Therefore, we propose an ILP formalization of the problem, that could be solved only for a small network. We also propose an approximation of the lower-bound overhearing based on simple graph coloring technique. We show that overhearing is the process which consumes the most energy in both small and large networks.

\section{REFERENCES}

[1] I. F. Akyildiz, W. Su, Y. Sankarasubramaniam, and E. Cayirci, "Wireless sensor networks: a survey," Computer Networks, vol. 38, no. 4, pp. 393 422,2002

[2] H. Kim, Y. Seok, N. Choi, Y. Choi, and T. Kwon, "Optimal multi-sink positioning and energy-efficient routing in wireless sensor networks," in ICOIN'05, 2005, pp. 264-274.

[3] L. Qiu, R. Ch, K. Jain, and M. Mahdian, "Optimizing the placement of integration points in multi-hop wireless networks," in in Proceedings of ICNP, 2004, p. 2004.

[4] E. I. Oyman and C. Ersoy, "Multiple sink network design problem in large scale wireless sensor networks," in Proceedings of the IEEE International Conference on Communications, ser. ICC 2004. Piscataway, NJ, USA: IEEE Press, 2009, pp. 3663-3667.

[5] L. B. Saad and B. Tourancheau, "Multiple mobile sinks positioning in wireless sensor networks for buildings," Sensor Technologies and Applications, International Conference on, vol. 0, pp. 264-270, 2009.

[6] Z. Vincze and R. V. andAttila Vidacs, "Deploying multiple sinks in multi-hop wireless sensor networks," Pervasive Services, IEEE International Conference on, vol. 0, pp. 55-63, 2007.

[7] C.-Y. Chang and H.-R. Chang, "Energy-aware node placement, topology control and mac scheduling for wireless sensor networks," Computer
Networks, vol. 52, no. 11, pp. 2189 - 2204, 2008. [Online]. Available: http://www.sciencedirect.com/science/article/pii/S1389128608001011

[8] G. Molina and E. Alba, "Wireless sensor network deployment using a memetic simulated annealing," in Applications and the Internet, 2008. SAINT 2008. International Symposium on, 2008, pp. 237-240.

[9] F. Baccelli and B. Blaszczyszyn, "Stochastic geometry and wireless networks, volume 1: Theory," Foundations and Trends in Networking, vol. 3, no. 3-4, pp. 249-449, 2009.

[10] — - "Stochastic geometry and wireless networks, volume 2: Applications," Foundations and Trends in Networking, vol. 4, no. 1-2, pp. $1-312,2009$.

[11] "Chipcon CC2420 Datasheet: http://focus.ti.com/lit/ds/symlink/cc2420.pdf, Texas Instruments," 2007. 